\title{
AN INVESTIGATION OF THE DIFFUSION ERRORS IN DIFFUSION VORTEX METHODS
}

\author{
M.-J. Huang * Y.-Y. Chen ${ }^{* *}$ \\ Department of Mechanical Engineering \\ National Taiwan University \\ Taipei, Taiwan 10617, R.O.C.
}

\begin{abstract}
To model the viscous effect, the core area of particles in Leonard's core spreading vortex method must grow linearly in time, which however results in growing convective errors and consequently causes the failure of a correct convergence of the method to the Navier-Stokes equations. The so-called diffusion vortex method was proposed and claimed to have smaller core-area growth rates because part of the viscous effect is modeled into the movement of particles. The growth rates however are non-uniform and an additional diffusion error arises due to the nonzero divergence of the diffusion velocity. The goal of this work is to analyze the associated errors of several existing versions of the diffusion vortex method and compare them with that of Leonard's. Simulations of two axisymmetric flows are performed to measure the involved diffusion errors and consequently distinguish these diffusion vortex methods. The results show that the circulation conservation is important, besides a small core-area growth rate, in obtaining a good accuracy. Under the consideration of both efficiency and accuracy, the diffusion vortex method in which each vortex particle conserves its own circulation on its core area alone is recommended.
\end{abstract}

Keywords : Vortex method, Diffusion velocity, Circulation.

\section{INTRODUCTION}

In solving two-dimensional incompressible flows, because of its compactness, the vorticity field is more efficient to be solved, compared to the velocity/pressure field. Among the various existing simulation methods, the discrete vortex method has been developed Lagrangian and grid-free. In a discrete vortex method [1], the convection of packets of vorticity (called vortex particles) is tracked by assuming that each packet moves as a whole at its central velocity. The deformation of the packet is ignored and the so-induced error is acceptable as long as the area covered by the packet of vorticity (core area) is small. Thereby, the explicit discretization of the convection terms and the associated stability constraint are avoided.

Viscous effects however stimulate the diffusion of vorticity. In 1980, Leonard [2] proposed the core spreading concept to account for the viscous effects. He found that the diffusion effect can be completely captured if each core area is modeled to grow linearly in time. This scheme is simple and efficient but the linear growth is too fast and no reasonably long time simulation is allowed due to the ignorance of the deformation mentioned above (Greengard, [3]). An alternative viscous treatment simply uses grid-based finite difference methods by mapping data between the Eulerian and Lagrangian grids, such as Chang et al [4].
Interpolations are therefore needed in order to evaluate the Laplacian and perform the mapping, which possibly results in excessive numerical diffusion. The random walk approach proposed by Chorin [5] added a pseudorandom velocity to the particle velocity. The scheme was shown to converge slowly and provide lowresolution, although being stable. The particle strength exchange scheme (Degond et al. [6]) redistributes the strengths (circulations) among vortex elements to account for diffusion by formulating a kernel that can evolve with the vorticity field. Fishelov [7] proposed to apply the Laplace operator on the convolution of some cut-off function with the delta distribution function. Difficulties are encountered however when the flow becomes strained in the last two methods.

The diffusion vortex method was first proposed by Ogami and Akamatsu [8] in 1991 and modified later by Kempka and Strickland [9], Shintani and Akamatsu [10], and Huang [11]. In this method, a diffusion velocity is defined and employed to model part of the viscous effect. The core-area growth rate is then adjusted to model the rest. This method is of interest because its growth rate is found indeed to be smaller than Leonard's. And it has a stronger physical basis in the sense that the circulation on any arbitrary "diffusion material surface" (a surface which is convected at the sum of the fluid velocity and the diffusion velocity) is conserved. To determine the core-area growth rates, there have been

\footnotetext{
*Associate Professor ** Graduate student 
many proposals. Unfortunately each of them requires lots of computations and cannot account for the viscous effect perfectly like Leonard's scheme does. Errors therefore arise from treating not only the convection terms but also the diffusion terms. The latter obviously distinguishes among the diffusion vortex methods.

In the present work, we attempt to do the discrimination. First, the correlations of errors with the core sizes are analyzed. Then flows, which are axisymmetric for the sake of highlighting the diffusion errors, are simulated for comparisons. A best choice will be suggested under the consideration of both accuracy and efficiency at the end. This paper is arranged as follows. The diffusion vortex methods to be investigated are introduced in Sec. 2. The error analysis is given in Sec. 3. These methods are then employed to simulate the evolutions of a single Burgers vortex and a vorticity disk in Sec. 4. Accuracies are compared there. Conclusion is given in Sec. 5.

\section{DIFFUSION VORTEX METHODS}

We consider a discretization of a given vorticity field $\omega(\vec{x}, t)$ in use of many Gaussian blobs as follows

$$
\omega(\vec{x}, t)=\sum_{j=1}^{N} \omega_{j}(\vec{x}, t)=\sum_{j=1}^{N} \frac{\Gamma_{j}}{\pi \sigma_{j}^{2}} \exp \left(-\frac{\left|\vec{x}-\vec{x}_{j}\right|^{2}}{\sigma_{j}^{2}}\right)
$$

where $\vec{x}_{j}(t), \Gamma_{j}(t)$ and $\sigma_{j}(t)$ are the location, strength (circulation), and core size of the $j^{\text {th }}$ vortex particle, which must evolve properly so that Eq. (1) fulfills the vorticity equation

$$
\frac{\partial \omega}{\partial t}+\vec{u} \cdot \nabla \omega=v \nabla^{2} \omega
$$

The velocity components $u$ and $v$ induced by these blobs can be computed by the Biot-Savart law to be

$$
\begin{aligned}
& u(\vec{x}, t)=-\sum_{j=1}^{N} \frac{\Gamma_{j}}{2 \pi} \frac{\left(y-y_{j}\right)}{\left|\vec{x}-\vec{x}_{j}\right|^{2}} \cdot\left(1-\exp \left(-\frac{\left|\vec{x}-\vec{x}_{j}\right|^{2}}{\sigma_{j}^{2}}\right)\right) \\
& v(\vec{x}, t)=+\sum_{j=1}^{N} \frac{\Gamma_{j}}{2 \pi} \frac{\left(x-x_{j}\right)}{\left|\vec{x}-\vec{x}_{j}\right|^{2}} \cdot\left(1-\exp \left(-\frac{\left|\vec{x}-\vec{x}_{j}\right|^{2}}{\sigma_{j}^{2}}\right)\right)
\end{aligned}
$$

In Leonard's core spreading scheme, the vortex particles are convected at the local fluid velocities and the core area grows linearly in time, namely,

$$
\begin{gathered}
\frac{d \vec{x}_{j}}{d t}=\vec{u}\left(\vec{x}_{j}, t\right) \\
\frac{1}{\sigma_{j}^{2}} \frac{d \sigma_{j}^{2}}{d t}=\frac{4 v}{\sigma_{j}^{2}}
\end{gathered}
$$

with $v$ being the fluid viscosity. On the other hand, the diffusion vortex method (DVM) splits the viscous effect into two parts and rewrites Eq. (2) as

$$
\frac{\partial \omega}{\partial t}+\left(\vec{u}-\frac{v \nabla \omega}{\omega}\right) \cdot \nabla \omega=\omega \nabla \cdot\left(\frac{v \nabla \omega}{\omega}\right)
$$

A diffusion velocity is thus defined as

$$
\vec{u}_{d} \equiv-\frac{v}{\omega} \nabla \omega=\frac{v}{\omega(\vec{x})}\left(\sum_{j=1}^{N} \frac{2\left(\vec{x}-\vec{x}_{j}\right)}{\sigma_{j}^{2}} \omega_{j}(\vec{x})\right)
$$

and used to model part of the viscous effect by forcing vortex particles moving at both of the fluid velocity and the viscous diffusion velocity:

$$
\frac{d \vec{x}_{j}}{d t}=\vec{u}\left(\vec{x}_{j}, t\right)+\vec{u}_{d}\left(\vec{x}_{j}, t\right)
$$

The rest of the viscous effect (the right hand side of Eq. (7)) is then treated through the adjustment of the vortex core size. Several ideas have been proposed. The first one ignores the right hand side of Eq. (7). That is, the core area remains unchanged in time (labeled VDM0) [8]. Later, investigators [9] noticed that if a surface $S$ moving at the sum of the fluid velocity and the diffusion velocity (called a "diffusion material surface" herein), its area is not conserved but its circulation is preserved, i.e.

$$
\begin{gathered}
\frac{1}{S} \frac{d S}{d t}=\int_{S} \nabla \cdot \vec{u}_{d} d s \\
\left(\frac{\partial}{\partial t}+\left(\vec{u}+\vec{u}_{d}\right) \cdot \nabla\right) \int_{S} \omega d s \equiv \frac{d \Gamma_{S}}{d t}=0
\end{gathered}
$$

The second VDM scheme (labeled "VDM1") thus views each core area as a circular diffusion material surface and chooses

$$
\frac{1}{\sigma_{j}^{2}} \frac{d \sigma_{j}^{2}}{d t}=\frac{1}{S_{j}} \int_{S_{j}} \nabla \cdot \vec{u}_{d} d s \equiv \overline{\left(\nabla \cdot \vec{u}_{d}\right)_{j}}
$$

where $S_{j}$ is the circular area centered at $\vec{x}_{j}$ of radius $\sigma_{j}$. The circulation $\Gamma_{j}$ is consequently conserved on the surface $S_{j}$ at all times.

The computation of the averaged divergence of the diffusion velocity is consuming however. The third version of the diffusion vortex method (labeled "VDM2" [9]) suggests replacing the surface average value by the value at the center. That is,

$$
\frac{1}{\sigma_{j}^{2}} \frac{d \sigma_{j}^{2}}{d t}=\left(\nabla \cdot \vec{u}_{d}\right)\left(\vec{x}_{j}\right)
$$

Since the vortex particles move at the velocity given by Eq. (9), the region they occupy constitute a diffusion material surface. Let $r_{j 1}$ be the distance between the $j^{\text {th }}$ particle and its nearest neighbor. If the diffusion material area represented by the $j^{\text {th }}$ particle is approximated to be proportional to $r_{j 1}^{2}$ (a square area of side $r_{j 1}$ ), then 


$$
\frac{1}{\sigma_{j}^{2}} \frac{d \sigma_{j}^{2}}{d t} \approx \frac{1}{r_{j 1}^{2}} \frac{d r_{j 1}^{2}}{d t}
$$

This is the method "VDM3" proposed by Shintani and Akamatsu [10].

Noticed is that actually none of the above four schemes fulfills the conservation constraint, Eq. (11). Because the core areas are generally overlapped, which is a critical factor for getting a smooth distribution by Eq. (1), ignorance of contributions to circulation on the surface $S_{j}$ from vortices other than $j$ may not be acceptable. The circulation conserved scheme (labeled "CCS" scheme [11]) therefore computes the true growth rates under the consideration of all possible contributions and an exact conservation of the circulations on the diffusion material surfaces $S_{j}$. For flexibility, the diffusion material surface $S_{j}$ is now redefined as the circular area centered at $\vec{x}_{j}$ of radius $\delta_{j}=\alpha \sigma_{j}$ where $\alpha$ is a free parameter. Now both the surface $S_{j}$ and the vortex particle $j$ are as usual assumed to move at the sum of the fluid velocity and the diffusion velocity at the center. The resulting governing equations for the growth rates consequently become

$$
\sum_{j=1}^{N} \frac{1}{\sigma_{j}^{2}} \frac{d \sigma_{j}^{2}}{d t} A_{i j}=\overline{\left(\nabla \cdot \vec{u}_{d}\right)_{j}} \sum_{j=1}^{N} D_{i j}-\sum_{j=1}^{N} \frac{1}{r_{i j}^{2}} \frac{d r_{i j}^{2}}{d t} B_{i j}
$$

where $A_{i j}$ and $B_{i j}$ are matrices accounting for the mutual effect of core-area changes and that of separating in between vortex particles; $D_{i j}$ accounts for the effect of expanding of the diffusion material surface itself. Detailed expressions of these matrices are as follows:

$$
\begin{gathered}
A_{i j}=\frac{\Gamma_{\mathrm{j}}}{\pi} \frac{\delta_{i}^{2}}{\sigma_{j}^{2}} F\left(\frac{r_{i j}}{\delta_{i}}, \frac{\delta_{i}^{2}}{\sigma_{j}^{2}}\right) \\
D_{i j}=\Gamma_{\mathrm{j}} \frac{\delta_{i}^{2}}{\sigma_{j}^{2}} I_{0}\left(2 \frac{r_{i j}}{\delta_{i}} \frac{\delta_{i}^{2}}{\sigma_{j}^{2}}\right) \exp \left(-\frac{\delta_{i}^{2}}{\sigma_{j}^{2}}\left(1+\frac{r_{i j}^{2}}{\delta_{i}^{2}}\right)\right)
\end{gathered}
$$

and

$$
B_{i j}=D_{i j}-A_{i j}
$$

where

$$
F(y, d)=\int_{0}^{\pi} f(y, \theta, d) d \theta
$$

and

$$
\begin{aligned}
f(y, \theta, d)= & \left.\left(1-y \cos \theta+y^{2} \sin ^{2} \theta\right) e^{-d(1-2 y \cos \theta)}-y^{2} \sin ^{2} \theta\right\} \\
& \cdot \exp \left(-d y^{2}\right) \\
+ & \sqrt{\frac{\pi}{d}} y \cos \theta\left(\frac{1}{2}-d y^{2} \sin ^{2} \theta\right) \exp \left(-d y^{2} \sin ^{2} \theta\right) \\
& \cdot\{\operatorname{Erf}((1-y \cos \theta) \sqrt{d})-\operatorname{Erf}(-y \cos \theta \sqrt{d})\}
\end{aligned}
$$

in which $I_{0}$ and $\operatorname{Erf}$ are the modified Bessel function of order zero and the error function respectively. Moreover, $\mathrm{B}_{i j}=0$ when $i=j$. Function values are tabulated and the interpolation is employed in simulations to reduce the computational amount.

A simple comparison among these diffusion vortex methods can be made by computing the instantaneous growth rates of two identical vortex particles separated by a distance $h$. Figure 1 shows the growth rates normalized by $4 v / \sigma^{2}$, that is, Leonard's growth rate. It shows that all VDM schemes except CCS have smaller growth rates than Leonard's. The difference between VDM1 and VDM2 is small, especially when $h / \sigma<1$. Replacing the divergence of diffusion velocity by the separating rate between particles (VDM3) seemingly is not proper. The CCS scheme employing an $\alpha=1$ has a growth rate greater than Leonard's when $h / \sigma>1.13$; smaller otherwise. The growth rates of CCS with different $\alpha$ 's are similar but increasing with decreasing $\alpha$. For most applications $h / \sigma<1$ is in use for obtaining a smooth vorticity field. Therefore, one may conclude that all VDMs including CCS have smaller core-area growth rates than Leonard's in general applications.

\section{ERROR ANALYSIS}

Two kinds of errors are discussed herein - the convection error and the diffusion error. By substituting Eq. (1) into Eq. (2), one finds

$$
\begin{aligned}
\frac{\partial \omega}{\partial t}+\vec{u} & \cdot \nabla \omega-v \nabla^{2} \omega \\
= & \sum_{j=1}^{N}\left(\vec{u}(\vec{x})-\frac{d \vec{x}_{j}}{d t}\right) \cdot \nabla \omega_{j}(\vec{x}) \\
& +\sum_{j=1}^{N} \omega_{j}(\vec{x})\left(\frac{\left|\vec{x}-\vec{x}_{j}\right|^{2}}{\sigma_{j}^{2}}-1\right)\left\{\frac{1}{\sigma_{j}^{2}} \frac{d \sigma_{j}^{2}}{d t}-\frac{4 v}{\sigma_{j}^{2}}\right\}
\end{aligned}
$$

The first term on the right hand side represents the (convection) error due to the inconsistency between the

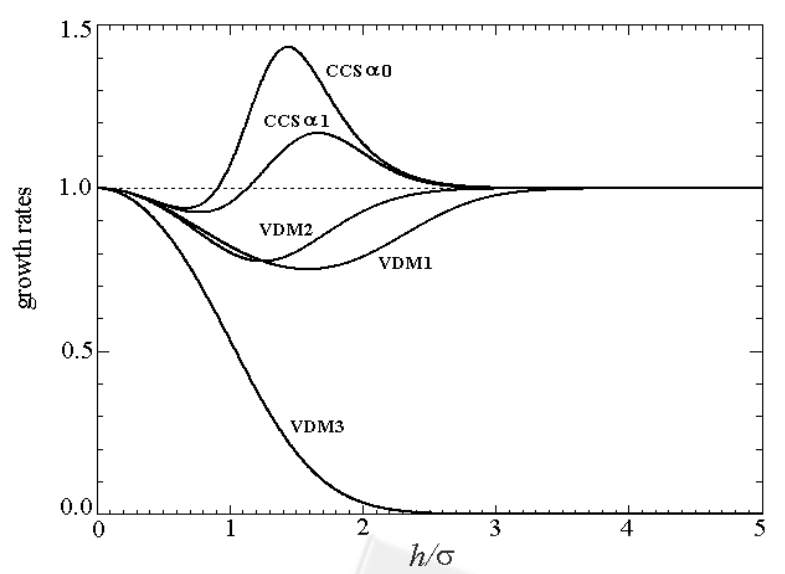

Fig. 1 The instantaneous growth rates normalized by $4 v / \sigma^{2}$ of two identical vortex particles separated by a distance $h$ 
non-uniform velocity distribution on the core area and the assumption that the vorticity blob $\omega_{j}$ moves as a whole at the central velocity, Eq. (5). This error can be shown to be on the order of $\sigma_{j}^{2}$ [2] by approximating it as

$$
\sum_{j}\left\{\left(\vec{x}-\vec{x}_{j}\right) \cdot \nabla\right\} \vec{u}\left(\vec{x}_{j}\right) \cdot \nabla \omega_{j}(\vec{x})
$$

where the summation is taken over vortex particles in the neighborhood of $\vec{x}$, and noticing that for a Gaussian blob $\omega_{j}(\vec{x})$,

$$
\left(\vec{x}-\vec{x}_{j}\right) \omega_{j}(\vec{x})=-\frac{\sigma_{j}^{2}}{2} \nabla \omega_{j}
$$

Therefore, the core sizes of vortex particles must remain small to keep a small convection error. The second term (diffusion error) is exactly zero if Eq. (6) is employed. Therefore, Leonard's growth rate accounts for the viscous effect completely. The linear growth rate is however too rapid, unless the fluid viscosity is very small.

Substituting Eq. (1) into Eq. (7), one can similarly obtain

$$
\begin{aligned}
\frac{\partial \omega}{\partial t}+ & \left(\vec{u}+\vec{u}_{d}\right) \cdot \nabla \omega+\omega \nabla \cdot \vec{u}_{d} \\
= & \sum_{j=1}^{N}\left(\vec{u}(\vec{x})+\vec{u}_{d}(\vec{x})-\frac{d \vec{x}_{j}}{d t}\right) \cdot \nabla \omega_{j}(\vec{x}) \\
& +\sum_{j=1}^{N} \omega_{j}(\vec{x})\left\{\left(\frac{\left|\vec{x}-\vec{x}_{j}\right|^{2}}{\sigma_{j}^{2}}-1\right) \frac{1}{\sigma_{j}^{2}} \frac{d \sigma_{j}^{2}}{d t}+\nabla \cdot \vec{u}_{d}(\vec{x})\right\}
\end{aligned}
$$

The convection error is second order accurate in $\sigma_{j}^{2}$ again, like that of Leonard's method:

$$
\begin{aligned}
& \sum_{j}\left[\left(\vec{x}-\vec{x}_{j}\right) \cdot \nabla\right]\left(\vec{u}+\vec{u}_{d}\right)\left(\vec{x}_{j}\right) \cdot \nabla \omega_{j}(\vec{x}) \\
& \quad \sim \sum_{j} \frac{\sigma_{j}^{2}}{2} \nabla \omega_{j}(\vec{x}) \cdot \nabla\left(\vec{u}+\vec{u}_{d}\right)\left(\vec{x}_{j}\right) \cdot \nabla \omega_{j}(\vec{x})
\end{aligned}
$$

As far as the diffusion error is concerned, VDM0 is zero-order accurate as it simply ignores the existence of $\omega \nabla \cdot \vec{u}_{d}$ and thus is discarded in the present study.

The diffusion error is second order in $\sigma$ for VDM2, which can be shown in a similar way like what we have done in treating the convection error to be

$$
\sum_{j=1}^{N} \frac{\sigma_{j}^{2}}{2} \nabla \omega_{j}(\vec{x}) \cdot\left\{\frac{\nabla \omega_{j}(\vec{x})}{\omega_{j}(\vec{x})} f\left(\vec{x}_{j}\right)-\nabla f\left(\vec{x}_{j}\right)\right\}
$$

where $f(\vec{x}) \equiv \nabla \cdot \vec{u}_{d}(\vec{x})$. So is VDM1 since

$$
\frac{1}{S_{j}} \int_{S_{j}} f(\vec{x}) d A \approx f\left(\vec{x}_{j}\right)+\frac{\sigma_{j}^{2}}{8} \nabla^{2} f\left(\vec{x}_{j}\right)
$$

Noticed is that VDM1 does not necessarily work better than VDM2 and it is because the core area $\pi \sigma^{2}$ is not a real diffusion material surface but just characterizes the region in which circulation of the $j^{\text {th }}$ particle is mainly distributed. The use of Eq. (12) is thus not necessarily true. As far as the circulation conservation is considered, VDM1 could be expected to be better if the overlapping were little.

The diffusion error involved with VDM3 may be explained in the following way. Basically, if the vortex particles are uniformly distributed in space, it is reasonable to say that each particle occupies or represents a diffusion material area of $r_{j 1}^{2}$. Equation (14) follows consequently and is second order accurate in $\sigma_{j}$ like VDM2. The uniformity is thus important for VDM3. Finally, the circulation preserving scheme, Eq. (15), is nothing more than nullifying the total error (the integral of the right hand side of Eq. (24)) over the disk $S_{j}=\pi\left(\alpha \sigma_{j}\right)^{2}$. If $\alpha=0$, zero net errors at particles' locations are shot then. While a better accuracy can thus be expected, CCS requires much more computations because a time-changing matrix equation needs solving.

In remark, the diffusion vortex methods have an extra diffusion error, besides a second order convection error. The core-area growth rates are really made smaller than those in Leonard's core spreading scheme. A longer time simulation can thus be expected.

\section{ACCURACY TEST}

While the convection errors are possibly reduced in diffusion vortex methods due to smaller core-area growth rates, the induced diffusion errors make differences and distinguish themselves. In the following subsections, the diffusion errors are measured and compared through simulations of two flows. These flows are chosen to be axisymmetric because then the convection error in Leonard's scheme is little because of no radial motion and because of the symmetry. Moreover, analytical solutions are also available.

\subsection{Burgers Vortex}

The first attempt is to simulate a decaying Burgers vortex with an initial characteristic radius of 0.1 and viscosity $v=1$. The initial distribution is discetized approximately into a superposition of 36 Gaussian blobs having a same initial core size $\sigma_{j}=1 / 30$, distributed along three rings of radii $1 / 30,2 / 30$, and $3 / 30$ as shown in Fig. 2. The particles' strengths $\Gamma_{j}$ are then adjusted so that the vorticity values are exact at particles' locations. Differences however are existent elsewhere. To avoid the contamination of the initial discretization errors, the analytical solution found by using the discretized initial vorticity distribution, which is at least nearly axisymmetric as seen from Fig. 2 (not perfectly because strengths of particles on a same ring are not all the same) [12], 


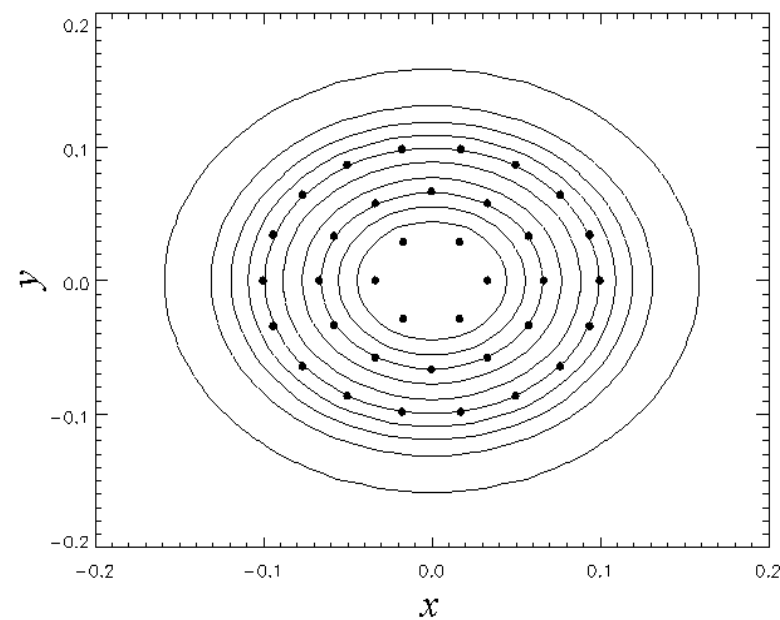

Fig. 2 The initial particles' locations and the vorticity contours constructed by these vortex particles

$$
\omega(\vec{x}, t)=\frac{1}{4 v t} \sum_{j=1}^{N} \int \omega_{j}\left(\vec{x}^{\prime}, 0\right) \exp \left\{-\frac{\left|\vec{x}-\vec{x}^{\prime}\right|^{2}}{4 v t}\right\} d \vec{x}^{\prime}
$$

is referenced. The simulations are time marched by the modified Euler method. The time increment $d t$ has been chosen to be very small (below $10^{-4}$ ) in order to highlight the spatial accuracy.

Figure 3 shows the measured root-mean-square errors, averaged over particles' locations. The schemes arranged according to their accuracies in ascending order are: VDM3 < VDM2 < VDM1 < CCS < Leonard. Not surprisingly Leonard's scheme performs best because it has no diffusion error at all. Although convection error is little in Leonard's scheme (the induced velocity $\vec{u}$ is only azimuthal), it does matter however in diffusion vortex methods because the diffusion velocity causes movements in the radial direction. Among the diffusion vortex methods, CCS performs best. It must be because it preserves the circulations on the selected diffusion material surfaces. VDM1 works better than VDM2 and VDM3 because only early-time simulations are performed and overlapping among core areas is not serious. In other words, particle' contribution to circulation on its own core area is still major. VDM3 performs worst in this example probably because particles are not many enough to represent "surfaces". Figure 4 shows the growing core sizes with time. The growth rates in descending order are: Leonard < CCS < VDM1 < VDM2 $<$ VDM3, which is consistent with the prediction of two-vortex system in Section 2. Putting together the two orders mentioned above, it tells that whether the circulation is conserved dominates over the core size effect in determining the accuracy of a diffusion vortex method.

The dependence on the value of $\alpha$ of the accuracy of CCS is shown in Fig. 5. A larger $\alpha$ does not always result in a better accuracy. A larger $\alpha$ does imply more overlapping between the interested diffusion material surfaces and consequently increases the difficulty in

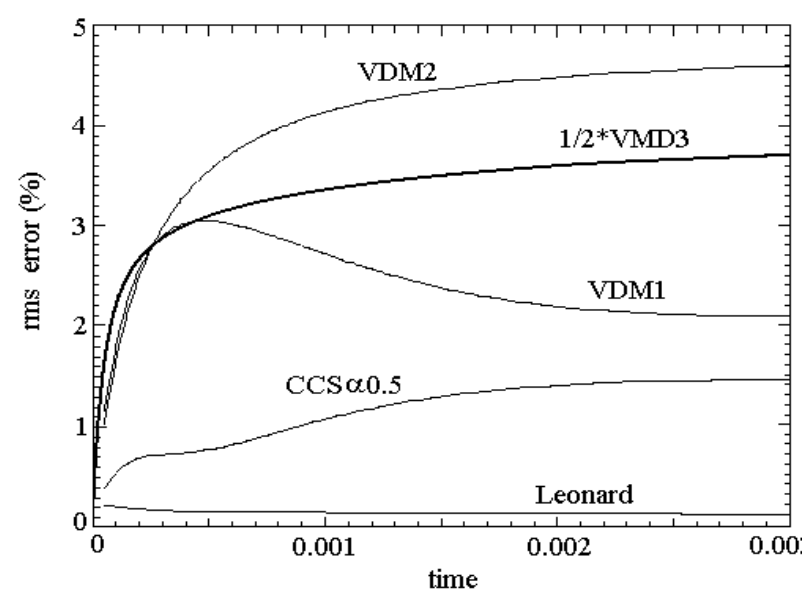

Fig. 3 The root-mean-square (rms) error averaged over particles' locations versus time. To put everything together, only half of the errors from VDM3 are plotted

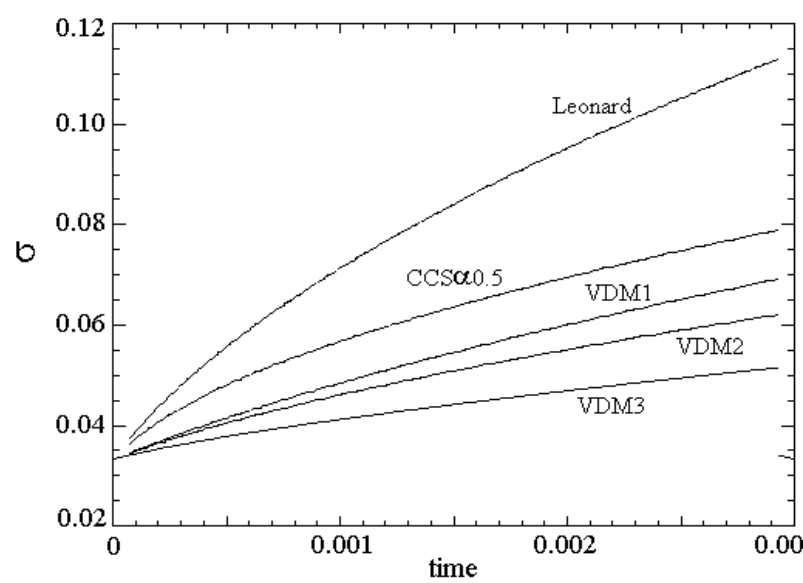

Fig. 4 The growing core sizes of one of particles versus time

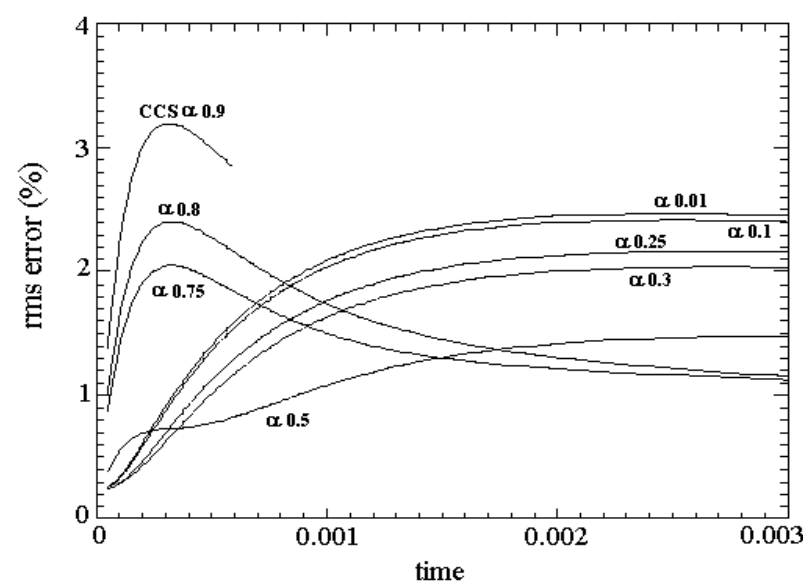

Fig. 5 The root-mean-square (rms) error averaged over particles' locations versus time from CCS with various $\alpha$ 's

conserving circulations on them at the same time. The numerical stability of CCS is found to decrease with increasing $\alpha$. It even blows up and simulations have to stop at some time no matter how small the time increment is chosen when $\alpha \geq 0.9$. The expanding 
rates of the core areas are found only weakly dependently on the value of $\alpha$ and therefore only the one with $\alpha=0.5$ is shown in Fig. 4 .

In remark, this test flow shows that a use of the viscous diffusion velocity is capable of reducing the expanding rates of the core areas compared to Leonard's method, which helps in lowering the convection errors, but generates additional diffusion errors. The preservation of circulation should be taken into consideration in controlling the total errors.

\subsection{Vorticity Disk}

We next simulate a vorticity field which is initially uniformly distributed within a disk of radius $R=1.5$ and zero outside. The total circulation is one and $v=1$. A total of 665 vortex particles having an initial $\sigma=0.1$ are employed and initially uniformly distributed within the disk as shown in Fig. 6. Again, Eq. (28) is employed to obtain the analytical solution because we don't really have discontinuity at $r=R$. Limited to the numerical instability, only the CCS with $\alpha=0$ is adopted and compared with all the other diffusion vortex methods.

The root-mean-square errors over the particles' locations are shown in Fig. 7. Noticed is the long-time accuracy. The performances are VDM1 < VDM3 < VDM $2<\mathrm{CCS}<$ Leonard. The accuracy of VDM1 is no longer superior to VDM2 and VDM3, because the core areas of particles are too large and overlapped lots. Mutual contributions to the circulations on core areas cannot be ignored any more. In that case, VDM2, whose computations are less and whose core-area growth rates are smaller, instead works better. VDM3 performs accidentally better than VDM1 at large times for no obvious reason. The growing core size of one of the most outer particles, which diffusion is most, is shown in Fig. 8. The core size from CCS is only about half Leonard's. Those from VDMs are even less. Most of all, VDM2 has a nice accuracy, reasonable computations, and smallest growth rates (good for controlling the convection error, if exists). These make it the best choice.

In Figs. 9 and 10 we show the vorticity radial distributions at $t=0.3$ and $t=3$. The solid line represents the analytical solution. Numerical distributions are represented by symbols which are also used to indicate (665) particles' instantaneous locations. At early times, the core areas of outer particles grow much faster and thus errors are larger there in all the diffusion vortex methods. At later times when all particles have large core areas, overlapping is more serious and consequently errors are bigger near the origin. This situation is worst in VDM1 as seen from Fig. 8 that it has a large core size and from Fig. 10 that data near the origin is more scattered, implying that the computed vorticity distribution is no longer axisymmetric. This problem however can be fixed if CCS is employed or if the circulation is numerically preserved.

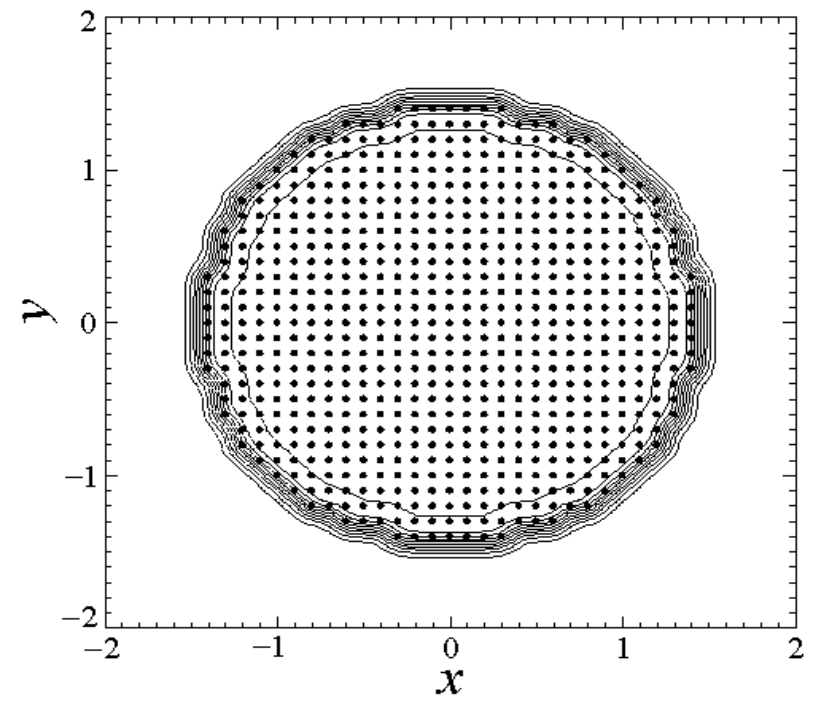

Fig. 6 The initial locations of 665 vortex particles and the initial vorticity contours

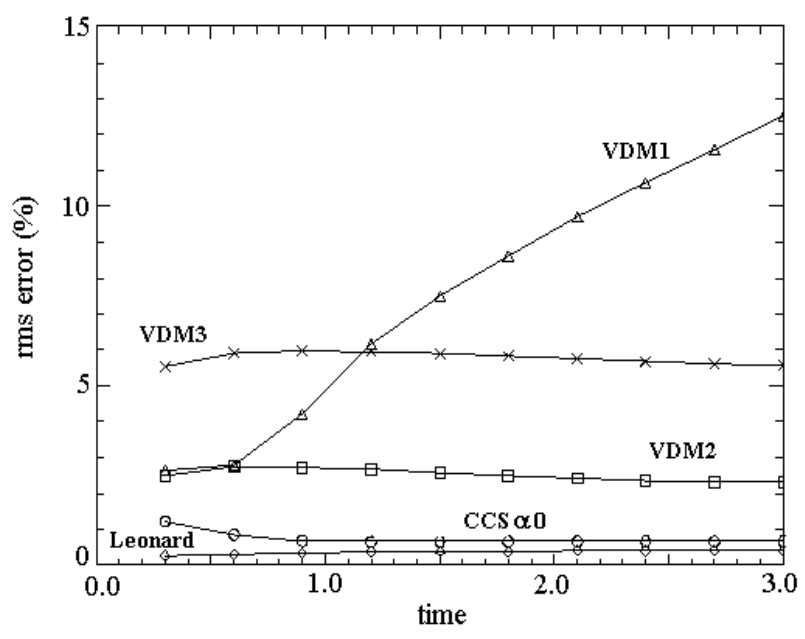

Fig. 7 The root-mean-square (rms) error averaged over particles' locations versus time

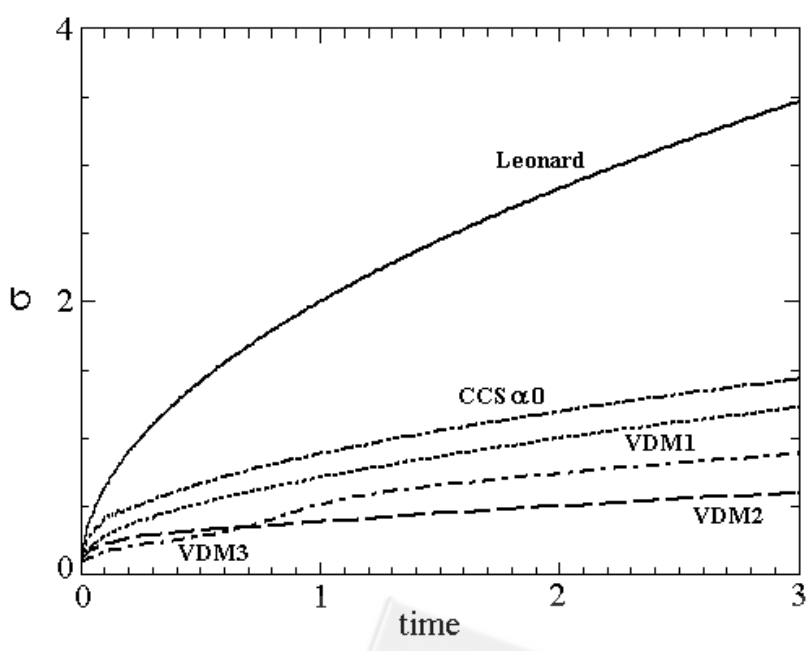

Fig. 8 The core size of one of the most outer particles versus time 

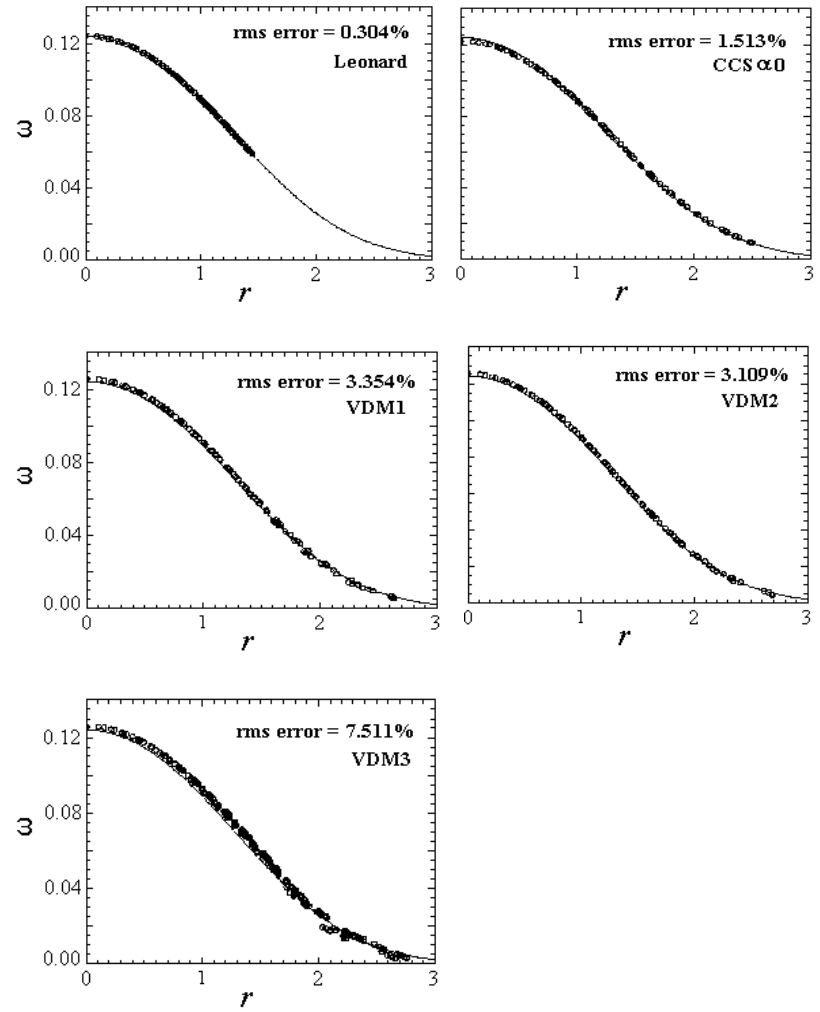

Fig. 9 Vorticity radial distribution at $t=0.3$. Solid line: exact; symbols: numerical
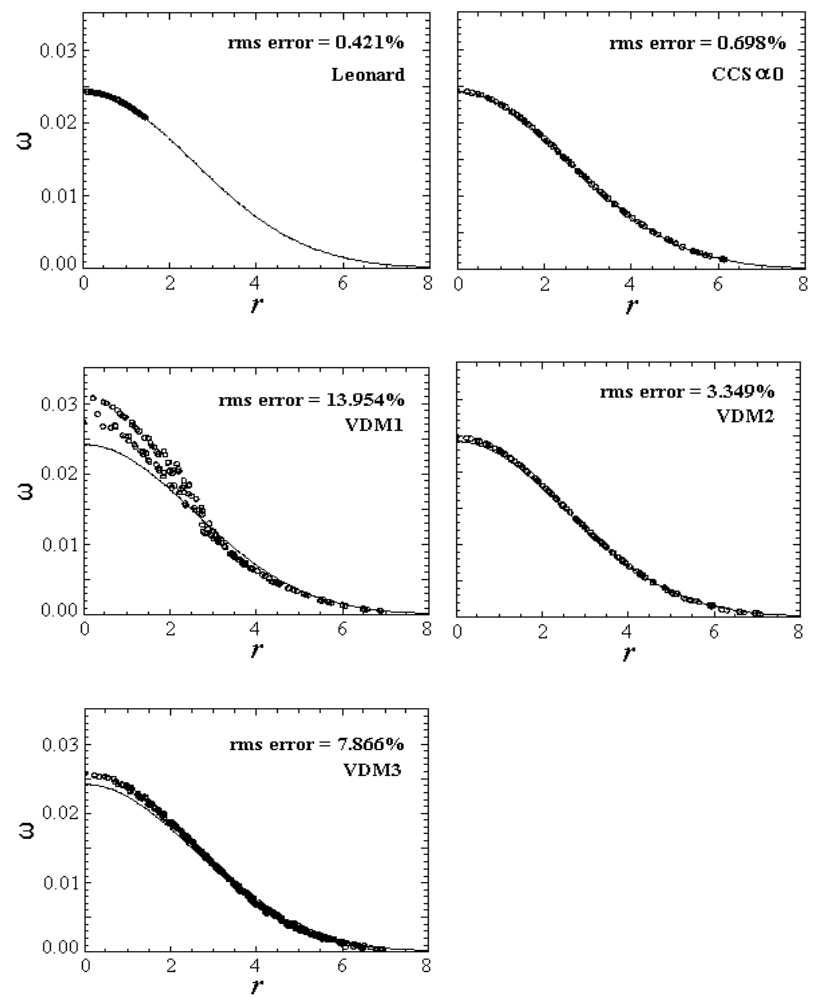

Fig. 10 Vorticity radial distribution at $t=3$. Solid line: exact; symbols: numerical

It is also worth mentioning that Leonard's particles have no diffusion velocity and thus stay within the disk forever. Vorticity diffuses outward through the expanding of particles' core areas. VDMs' and CCS' particles on the other hand move outward gradually by the diffusion velocity, and thus particles' locations can characterize properly the regions with non-negligible vorticity. This is a big advantage of the diffusion vortex methods. However only CCS maintains a good accuracy comparable to Leonard's at all times.

\section{CONCLUSION}

The lumped movements of vortex particles cause the so-called convection error, which is second order accurate in the core sizes of particles. Leonard's core spreading method has a linear growth rate and generally is too fast to achieve a long-time simulation. The diffusion vortex method splits the viscous effect into two parts - one accounted by moving particles at the diffusion velocity in addition to the fluid velocity and the other by the growth of core areas. The resulting growth rate is thus reduced with the bonus that particles' locations can also characterize regions with nonnegligible vorticity. A new error - diffusion error however is generated due to the fact that the diffusion velocity is not solenoidal, and is also second order accurate in the core size. To be better, this new error must be well controlled.

Theoretically in a diffusion vortex method, the circulation on any "diffusion material surface," a surface moving at the sum of the fluid velocity and the diffusion velocity, must be conserved. The circulation conserved scheme (CCS) attempts to do this and so does acquire a good accuracy. Its computational amount however is large and it must encounter the problem of numerically instability. VDM1 ignores mutual contributions and only preserves each particle's circulation on its own core area. When overlapping of core areas is much, VDM1 fails. VDM2 further simplify the computation of the surface-averaged growth rate by the value at the center of the core area. Its accuracy turns out to be nice, its computational amount is acceptable, and its core-area growth rates are small. VDM2 is thus recommended. Finally the approximation of core-area growth rate by the separation rate of nearby particles (VDM3) is not suggested.

\section{ACKNOWLEDGEMENT}

The support of this work by the National Science Council, Taiwan, R.O.C. under contract NSC 92-2212E-002-042 is gratefully acknowledged.

\section{REFERENCES}

1. Cottet, G.-H. and Koumoutaskos, P., Vortex Methods: Theory and Practice, Cambridge Univ. Press, Cambridge, UK (1999). 
2. Leonard, A., "Vortex Methods for Flow Simulations," J. Comput. Phys., 37, pp. 289-335 (1980).

3. Greengard, C., "The Core Spreading Vortex Method Approximates the Wrong Equation," $J$ Comput. Phys., 61, pp. 345-348 (1985).

4. Chang, C. and Chern, R., "A Numerical Study of Flow Around an Impulsively Started Circular Cylinder by a Deterministic Vortex Method," $J$. Fluid Mech., 233, p. 243 (1991).

5. Chorin, A. J., "Numerical Study of Slighly Viscous Flow," J. Fluid Mech., 57, pp. 785-796 (1973).

6. Degond, P. and Mas-Gallic S., "The Weighted Particle Method for Convection-Diffusion Equations, Part 1: The Case of an Isotropic Viscosity," Math. Comput., 53, pp. 485-507 (1989).

7. Fishelov, D., "A New Vortex Scheme for Viscous Flow," J. Comput. Phys., 86, pp. 211-224 (1990).

8. Ogami, Y. and Akamatsu, T., "Viscous Flow Simulation using the Discrete Vortex Model — The Diffusion Velocity Method," Computers and Fluids, 19, pp. 433-441 (1991).
9. Kempka, S. N. and Strickland, J. H., "A Method to Simulate Viscous Diffusion of Vorticity by Convective Transport of Vortices at a NonSolenoidal Velocity," Sandia Report, Sandia National Lab., SAND93-1763.UC-700 (1993).

10. Shintani, M. and Akamatsu, T., "Investigation of Two Dimensional Discrete Vortex Method with Viscous Diffusion Model," Computational Fluid Dynamics, 3, pp. 237-254 (1994).

11. Huang, M. J., "Circulation Conserved Diffusion Vortex Method," Transactions of the Aeronautical and Astronautical Society of the Republic of China, 35, pp. 65-72 (2003).

12. Haberman, R., Elementary Applied Partial Differential Equations: With Fourier Series and Boundary Value Problems, Prentice-Hall Inc. (1983).

(Manuscript received June 3, 2004, accepted for publication January 4, 2005.) 